\title{
The sick lobe hypothesis, field cancerisation and the new era of precision breast surgery
}

\author{
Mona P. $\operatorname{Tan}^{1}$, Tibor Tot $^{2}$ \\ ${ }^{1}$ Department of Breast Surgical Oncology, MammoCare, Singapore, Singapore; ${ }^{2}$ Department of Pathology \& Head, Pathology and Cytology \\ Dalarna, County Hospital Falun, Falun, Sweden \\ Contributions: (I) Conception and design: All authors; (II) Administrative support: None; (III) Provision of study materials or patients: None; (IV) \\ Collection and assembly of data: None; (V) Data analysis and interpretation: None; (VI) Manuscript writing: All authors; (VII) Final approval of \\ manuscript: All authors. \\ Correspondence to: Mona P. Tan, FRCS (Ed). Department of Breast Surgical Oncology, MammoCare, Singapore, 38 Irrawaddy Road, \#06-21, \\ Singapore 329563, Singapore. Email: jabezhopems@gmail.com.
}

\begin{abstract}
Understanding the ductal anatomy of the breast provides insights into tumorigenesis, which in turn offers guidance on therapeutic decisions. In this regard, the sick lobe hypothesis, which states that cancer arises from genetically unstable cells through mutations acquired in utero, forms the basis of malignant transformation. These 'at risk' cells line the mammary ductal-lobular system of a single 'sick' lobe and when exposed to noxious events in the surrounding microenvironment, further genetic changes occur which completes conversion to malignancy, in certain defined patterns. This review explores how anatomy, pathology and genomics can merge, not only to guide optimum surgery, but also to provide a more comprehensive portal for precision medicine.
\end{abstract}

Keywords: Breast cancer; sick lobe theory; field cancerisation; breast conservation treatment (BCT); multifocal/ multicentric breast cancer; precision breast surgery

Submitted Feb 15, 2018. Accepted for publication Sep 17, 2018.

doi: 10.21037 /gs.2018.09.08

View this article at: http://dx.doi.org/10.21037/gs.2018.09.08

\section{Introduction}

Sir Astley Cooper, a 19th century English anatomist and surgeon, provided one of the earliest insights into the anatomy of the breast (1). In his cadaveric studies, nipple orifices of mammary glands were injected with coloured wax, which demonstrated the lobar distribution of the ductal system (2). It appears that this knowledge of the delicate arrangement of ducts and lobules lay dormant with the progress of surgical therapeutics for breast cancer, for at the turn of the twentieth century, William Halsted promulgated the use of the radical mastectomy for this purpose (3). Due to the rudimentary understanding of the pathology and biology of breast cancer in the last century, coupled with the absence of screening to allow its early detection, mastectomy remained the mainstay of the treatment of breast cancer for at least seven decades. Then, in the last quarter of the twentieth century, Veronesi et al. and Fisher et al. ushered in a new epoch in the surgical treatment of breast cancer when they introduced the concept of breast conservation treatment (BCT) $(4,5)$. However, surgical diminution and pathology advancement moved in divergent trajectories, until more recently, when the work of Cooper is revisited and recaptured in our understanding of tumourigenesis.

Concurrent with the introduction of BCT, several pathology teams detected the presence of non-clinically evident malignant foci separate from the index cancer, and concluded that this posed a potential barrier to the efficacy of BCT $(6,7)$. It was noteworthy that this work failed to take into account Cooper's seminal work on breast anatomy. While certain facts were not immediately obvious when BCT was first introduced, accumulation of knowledge over time has made us cognisant that pathology of the breast 
needs to be studied in conjunction with anatomy for an improved understanding of carcinogenesis, and integrated with therapeutic strategies.

Prospective randomised controlled trials (RCTs) have demonstrated that breast conserving surgery (BCS) with radiotherapy confers non-inferior outcomes to mastectomy $(4,5)$. Some contemporary retrospective studies suggest that BCT may be superior to mastectomy in terms of survival and local control (8-15). Although there is potential for confounding, the evidence is substantial enough to question the basis for these outcomes. Part of the answer lies in the combination of the centuries-old knowledge of anatomy and modern understanding of mutagenesis.

\section{Embryology of the mammary gland \& the sick lobe hypothesis}

Mammary development commences in the 5 th week of intrauterine life identifiable by an ectodermal thickening from the axilla to the groin. This then condenses, during the 6th to 7 th week, to a single pair of mammary crests on the thorax between the 4th \& 5th intercostal space $(16,17)$ Primary mammary bud development ensues, with epithelial outgrowths penetrating downwards into the deepest of three layers of mesenchyme, then burgeoning into secondary buds between the 12 th and 21 st weeks. Thereafter, 15-20 radial outgrowths permeate the developing breast. These epithelial growths acquire a lumen lined by two layers of cuboidal cells, with epithelial and myoepithelial/ basal layers. The forming lactiferous ducts converge to open into a shallow mammary pit, which transform into a nipple during infancy. At birth, the gland may consist of a series of blind-ended tubes with bulbous tips (16), to well defined lobules and terminal duct lobular units, arranged radially in 15-20 lobes, draining through lactiferous ducts into ampullae (16), Further branching and terminal lobule development continues for the first 2 years of life (17), and is relatively quiescent until thelarche. At puberty, under the influence of ovarian oestrogens, the mammary ductal epithelium and surrounding stroma proliferate. This leads to the concomitant proliferation of collecting ducts, terminal duct lobular units, breast buds, vascular and connective tissue elements. Further maturation occurs during pregnancy where functional differentiation of the glandular parenchyma takes place to prepare the lobule-alveolar structure for lactation. Throughout the development and maturation process, the radial organisation of the mammary lobes is retained $(18,19)$.
It has been recognised for some time that duct anatomy is relevant to the neoplastic process (20). Ductal carcinoma in situ (DCIS) and lobular carcinoma in situ (LCIS) were found to occupy volumes of breast tissue which broadly conform to the catchment patterns of breast ductal territories. This is also consistent with the finding of the multifocal, multicentric and diffuse nature of breast carcinomas in a significant proportion of cases (21). Large section, or subgross histology studies has provided insight into the distribution of breast carcinoma. The sick lobe theory propounded by Tot postulates that fundamentally, breast carcinoma is a lobar disease (21). It is speculated that genetic instability is acquired through mutation during embryonic development, and the progenitor cells transmit this characteristic to their progeny along the ductal-lobular system occupying the entire lobe through pubertal development and maturation (22). Further noxious cellular or stromal events contribute to more mutations, with the sum of these factors leading to eventual malignant transformation. Since the cells with vulnerable genetic alterations are found within the same ductal tree, simultaneous or asynchronous multiple tumour foci originate from within a single lobe of the breast (23).

Anatomic studies using a software modelling programme on subgross pathology sections have demonstrated that the branching skeleton of a complete duct system takes on a pyramidal configuration with the summit at the nipple, consistent with the reported structure of a single sick lobe $(24,25)$. The duct systems within one breast appear to be independent of the others, and anastomoses between each ductal unit have not been consistently identified (24). The radial lobes associated with the individual duct systems vary significantly in size and may overlie one another. Malignant transformation within the 'sick lobe' generally occurs in three patterns. Involvement of a single terminal ductal-lobular unit (TDLU) or a segmental duct together with its branches and terminal units, is termed 'unifocal' or 'segmental'. The growth pattern referred to as 'multifocal' or 'peripheral' occurs when several distant TDLUs within the same lobe are affected, with uninvolved breast tissue in between. The third configuration is 'diffuse' where larger ducts are involved, forming the lobar pattern (25). Multicentric disease involves more than one lobe with variations of segmental, peripheral or diffuse patterns. These growth patterns are best evidenced in the in situ and early invasive phases of malignancy. The invasive components, if any, are small at this tumour stage, but the involved volume of the breast tissue (the extent of 
the disease) varies, as multiple tumour foci may develop simultaneously or the process may diffusely involve large parts of the sick lobe from the beginning $(25,26)$. The molecular evolution of breast cancers supports this theory, where similar genetic alterations are demonstrated in both precursor lesions and subsequent malignant tumours (27). This phenomenon is also demonstrated in the progression from papilloma to DCIS and at any one time, a spectrum of disease involving the ductal tree of one or more lobes is seen. Ultimately, these findings return us to the original concept of an 'at-risk' cell population distributed along the arborisation of the ductal system of a single lobe, likely to be acquired during formation of the breast in utero, which continue to exist through further quiescent and developmental stages.

With respect to multiple synchronous unilateral lesions of invasive breast cancer, Desmedt et al. analysed genetic mutations in homogenous phenotypic ductal multifocal lesions in terms of grade, oestrogen receptor (ER) and human epidermal growth factor receptor 2 (HER2) (28). Three 'genomic' groups were identified. Thirty-one percent of tumours were referred to as the 'homogeneous' group where all multifocal lesions carried the same mutations. The second or 'intermediate group', comprising $36 \%$ of patients, had both common and private mutations. The third group of patients had no single mutation in common among all samples from the investigated lesions. This last group was categorised as 'heterogeneous'. The only association of significance between inter-lesion heterogeneity and clinico-pathological characteristics was that of inter-lesion distance. It was noted that the lesions from patients of the heterogeneous group were further apart from each other than those from patients of the homogeneous group. This observation supports the concept of the sick lobe(s), where a greater degree of homogeneity of mutations in lesions in closer proximity is indicative of similar embryologic origin, while molecular heterogeneity in more distant tumour foci suggests that they are derived from separate ductal-lobular trees with different genetic ancestry. The logical implication of these findings is that surgical resection should take into account both the anatomic architecture and genetic distribution of the affected lobes.

The sick lobe hypothesis proposes the most probable basis of mammary carcinogenesis. However, another plausible explanation for tumorigenesis is the expansion of preneoplastic cell populations along defined anatomical spaces as genetic mutations are acquired, developing survival advantage through resistance to apoptosis and other cellular characteristics necessary for clonal expansion and replacement of normal adjacent cells.

\section{Field cancerisation and surgical resection volumes}

The understanding of carcinogenesis has advanced considerably with the dawn of the genomic era. Based on the study of genomic alterations, the concept of field cancerization on the basis of a genetic model has emerged (29). This proposed sequential, multi-step genetic model of carcinogenesis begins with a cell acquiring one or more genetic or epigenetic aberrations which allow it a proliferative advantage. It then proceeds to form a clonal field of similar cells without altering tissue architecture. The cells within this precursor field then amass more alterations, now creating phenotypic differences among clonal populations. With increasing genetic alterations favouring greater proliferative potential, one or more cells acquire sufficient traits for malignant conversion. The model implies that cells which have undergone early genetic events but have not acquired all the changes necessary for tumorigenesis constitute the field cancerised tissue and is entirely consistent with the sick lobe hypothesis. Leyba et al. have provided data suggesting that phenotypically normal epithelial tissue bearing 'hallmarks of cancer' are detected adjacent to breast tumours (29). These abnormalities, comprising certain molecular characteristics associated with cancer cells, are found in tissues $1 \mathrm{~cm}$ from tumour, but not in tissues $5 \mathrm{~cm}$ from tumour. This could imply that the histologically normal tissue $1 \mathrm{~cm}$ from the tumour could fall within the same segment, while parenchyma more than $5 \mathrm{~cm}$ belong to a different lobe. If so, these factors could be used as markers for adequacy of tissue resection.

The molecular characteristics of neighbouring histologically similar tissue and the microenvironment of the surrounding stroma may have implications for volume of surgical resection. Genomic instability, telomerase expression, epithelial to mesenchymal transition in epithelial cells, coupled with dense disorganised extracellular matrix and myofibroblasts are thought to be drivers of tumour initiation and progression (29). Hence, the amount of genetically affected tissue which remains in the breast could have bearings for tumour recurrence. If so, excision of such morphologically normal but molecularly 'primed' tissue is essential for local control. The combined extent of the affected ductal-lobular tree (sick lobe) and surrounding stroma can be considered to be the involved 'segment', 
which logically leads to the method of determining an adequate resection volume. Edgerton et al. demonstrated that tumour morphology and characteristics, viewed as histological surrogates for mechanistic parameters, allow the derivation of a mathematical model to predict tumour volumes and therefore inform extent of surgical resection (30). The anatomy of the mammary gland is derived from a common embryology and framework, although each individual has a unique architecture of duct arborisation. As such, the actual geometry and local extension of intraductal tumour cells may vary from patient to patient. Using a composite of pathologic characteristics and imaging, a formula is used to calculate the distribution of disease for surgical planning. Ultrasound has been shown to be useful in this respect (31), while it is postulated that magnetic resonance imaging (MRI) may also have some applicability. It is thought that mammography which identifies calcifications may not be as reliable (30). The proposed model by Edgerton et al. assumes a resection volume in the shape of an ellipsoid, which can be viewed to be concordant with the sick lobe theory. This is in contradistinction to other recommended surgical approaches, which prescribe a circular, or spherical resection volume around the tumour (32). From a surgical perspective, two flaws can be identified with these 'rounded' surgical techniques. The first, being that it fails to conform to the patterns of tumour progression and dissemination which has been independently validated by work in different settings and geographical locations. Secondly, a spherical configuration of resection acts as a strut preventing direct parenchymal closure, requiring more complicated procedures to fill the defect created by tumour resection (32). An elliptical resection not only optimises resection of the 'sick lobe', it leaves a resection defect which can be closed by the simpler manoeuvre of full thickness parenchymal flap mobilisation and direct closure (33). The latter procedure, which may be viewed as 'standard breast conservation surgery'(sBCS) optimises cosmetic outcomes without increasing risk of surgical complications $(34,35)$.

\section{Implications for BCT}

The seminal work by Veronesi et al. and Fisher et al. $(4,5)$ ushered in the era of BCT, the archetypal form of surgical de-escalation. The advent of screening mammography resulted in a greater proportion of women being diagnosed with early breast cancer, who are suitable candidates for BCT. While the early RCTs reported equivalent survival outcomes, there is, more recently, contemporary evidence that BCT may be associated with higher survival compared with mastectomy (8-12). It is therefore now crucial to optimise BCT rates in any population of patients diagnosed with breast cancer. However, local control does have an impact on overall survival and it is appropriate that ongoing research identifies correct selection criteria for best local control. This calls for a balance between adequate margins and overtreatment. The embryology, anatomy of the breast, the concepts of tumour progression and field cancerisation appear to merge in complete congruity with the sick lobe hypothesis, converging on a predicted resection volume conforming to an elliptical shape. Current data implies that small volumes of sick lobe retained in breast can be effectively treated by radiotherapy (RT) and/or systemic treatment. Still, further refinements may be possible through precision pathology, surgery and adjuvant therapy. Molecular profiling has enabled patient selection for deescalation of medical treatment and may play a role in RT too, as a genome-based model for adjusting radiotherapy dose is in its preliminary stages of evaluation (36).

The sick lobe hypothesis applies to the early phases of breast cancer development, especially in relation to the in situ phase when the malignant cells are able to maintain the ductal-lobular architecture and the basement membrane retains its integrity separating the tumour from the surrounding stroma. This configuration continues to exist in early invasive cancer but may be disrupted in more advanced invasive disease. There is yet no consensus for the definition of 'early breast cancer', but it has been suggested that pure in situ cancer, microinvasive carcinoma $(<1 \mathrm{~mm})$ and invasive cancers smaller than $15 \mathrm{~mm}$ may be considered in this category (26). It is essential that preoperative assessment takes the combined distribution, or aggregate growth pattern, of both invasive and in situ parts of the tumour for appropriate case selection and operative approach. This involves treatment planning based on consolidated clinical information from pathology and imaging, primarily using mammography and sonography. Such surgical resection mapping may be viewed as the forerunner of precision surgery. Although there is potential for MRI in preoperative mapping, the low specificity and changes in breast parenchymal shape in the prone position during imaging and supine position during surgery needs to be accounted for prior to its application for this purpose.

Precision surgery, where resection volume is based on the integration of imaging, tumour pathology and molecular characteristics of the microenvironment, is not yet a widely 
Table 1 Treatment options by aggregate (in situ \& invasive) tumour distribution categories, according to the sick lobe theory

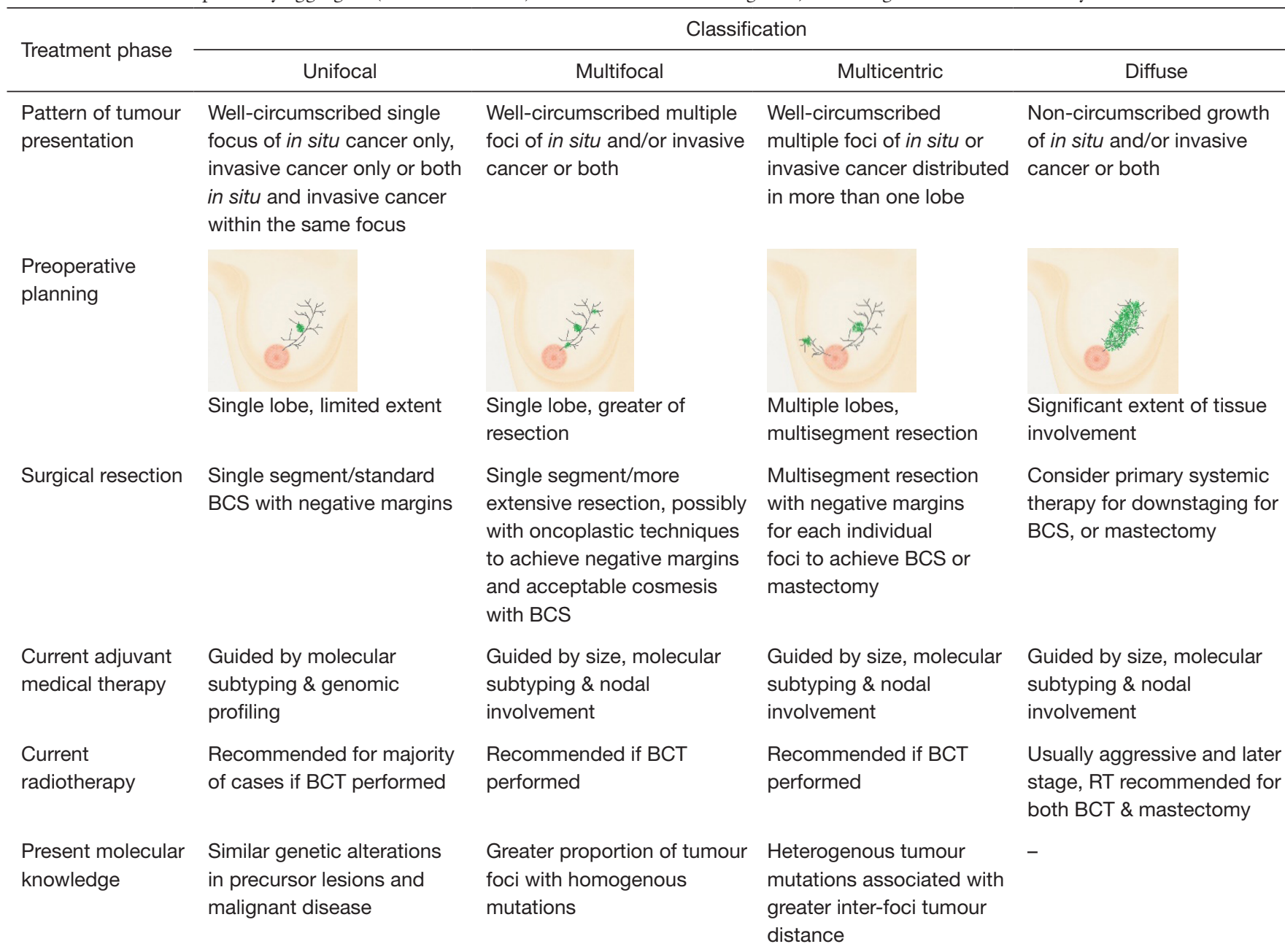

Future possibilities Molecular characteristics of epithelial cells in sick lobe or cancerized field may inform surgical margins, provide prognostic and predictive information for medical therapy \& radiotherapy

$\mathrm{BCS}$, breast conservation surgery; $\mathrm{BCT}$, breast conservation treatment.

embraced concept. The current state of the art offers the promise of its realisation for individualised treatment in the very near future. As it stands, contemporary knowledge of pathology and tumour biology offers adequate information to allow the classification of breast cancer distribution into four categories for optimal selection of surgical approach (Table 1) Unifocal cancer may be treated with sBCS, adhering to the elliptical resection format. These cancers are usually smaller, with better prognostic features. Molecular profiling allows further individualised systemic therapy. Multifocal tumours, defined as malignancies originating from multiple TDLUs distant from each other but within a single lobe can be surgically resected with a larger volume to excise as much of the 'sick lobe' as possible. However, a total extent of $4 \mathrm{~cm}$ may be a limiting factor for BCT, as this has implications for local control $(23,25)$. Multicentric breast malignancies, or those affecting different lobes simultaneously can be approached using a multisegment tissue resection pattern to excise the affected sick lobes (37). Finally, diffuse cancers, may be treated with mastectomy if they are in excess of $4 \mathrm{~cm}$ in size (23), or undergo primary systemic treatment for downstaging (38). Complete pathological response to neoadjuvant chemotherapy is a good prognostic indicator, and may allow for BCT in women with locally advanced breast cancers at presentation. The latter tumour categories 
(multifocal, multicentric and diffuse) may benefit from the biomarkers associated with field cancerisation more than unifocal tumours as they are known to be associated with more aggressive features $(39,40)$. and possibly higher risk of local recurrence. Molecular characteristics may be used to direct extent of surgical resection but it is essential to bear in mind that intratumoral and intertumoral heterogeneity exists in about $10-12 \%$ of patients with breast cancer (41). Clonal evolution leads to intratumoral biological variation in unifocal or diffuse lesions which in turn drives tumour behaviour and dissemination to lymph nodes (42). For multifocal/multicentric disease, intertumoral heterogeneity may have implications for survival. Hence, careful pathologic assessment is critical in therapeutic decision-making. Further research is needed to elucidate the optimum surgical approach and tailor treatment based on these characteristics. Until then, there may be value to attempting BCT even in these challenging circumstances at present, for it is speculated that a depressed immune system after more extensive surgery, combined with the absence of RT, could contribute to poorer survival outcomes $(43,44)$.

In summary, the knowledge of anatomy which was made available two centuries ago has provided, and continues to offer, clues to tumour distribution and pathology, which in turn may be used to inform surgical resection. The objective of surgery is the adequate resection of unstable 'sick lobe', preferably through BCT, as minimising stress to immune system with less extensive surgery appears to be associated with improved survival outcomes. The optimal operative approach currently involves an integration of disciplines of pathology, radiology, surgery and oncology (45), but genomics may play an increasing important role in the future.

\section{Future directions}

Independent pathologic and genetic studies performed in various geographic locations have provided evidence of a common thread between anatomy of the breast, the notion of field cancerisation and the sick lobe theory. There is evidence to support the following: that TDLUs arise from a single stem cell (27), an inverse relationship was demonstrated between similarities in tumour genetics and inter-foci distance (28), and tumour related mutations were independently detected in greater frequency closer to the tumour than tissue further away (29). These three phenomena are consistent with the concept of unstable cells distributed in the arborisation of ducts belonging to a single mammary lobe. Stromal changes in the proximity of the ductal-lobular tree may be drivers of tumour progression, or signifiers of prognosis. The presence of tumour infiltrating lymphocytes is associated with higher rates of complete pathologic response with neoadjuvant chemotherapy, which in turn indicates better survival outcomes (46). The postulated synergistic effect of immune response may not be limited to primary medical therapy, but may be extended to surgery as well. It has been suggested that less extensive procedures result in a lower degree of immune disruption and hence better survival outcomes $(43,44)$. All these concepts segue into a unifying hypothesis of carcinogenesis manifesting as a series of dynamic molecular aberrations occurring within a segment of breast parenchyma, which needs to be surgically excised adequately, rather than extensively, for optimal control. Pathologic and genomic examination of the resected tissue then informs adjuvant therapy. This sets the stage for further research to identify the appropriate imaging modalities for preoperative surgical planning, the optimum volume of tissue excision, and the significance of tumour related molecular abnormalities within histologically normal tissue in the margins of the resected specimen.

It is envisaged that the ideal future therapeutic approach is a multidisciplinary one. The pathologist takes a leading role by providing phenotypic and genomic details of tissue after the initial biopsy. This allows understanding of tumour characteristics as well as an estimation of proliferative pressure. This data, coupled with preoperative imaging assesses tumour volume, and is then entered into a software system, taking into account patient mammary volume and habitus. Using a mathematical model, optimum surgical resection volume can be calculated and a road map provided preoperatively for oncological resection adequacy and cosmetic outcome. A three-dimensional matrix may be generated and images superimposed on the patient to assist the surgeon during surgery. The tissue that is excised can then be further analysed for targeted adjuvant medical and radiation therapy $(36,47)$. This completes the full cycle for using pathology and genomics to tailor treatment based on the unique characteristics of both tumour and patient.

The insights offered by the sick lobe hypothesis, which has been hitherto under-recognised, may prove to be invaluable if future research is able to exploit its potential to identify selection criteria for precision surgery, medical treatment and RT in the treatment of breast cancer. 


\section{Acknowledgements}

None.

\section{Footnote}

Conflicts of Interest: The authors have no conflicts of interest to declare.

\section{References}

1. Cooper AP. The anatomy of the breast. London: Longman, Orme, Green, Brown and Longmans, 1840.

2. Cooper AP. Plates of the anatomy of the breast. London: Longman, Orme, Green, Brown and Longmans, 1840.

3. Halsted WS. The Results of Operations for the Cure of Cancer of the Breast Performed at the Johns Hopkins Hospital from June, 1889, to January, 1894. Ann Surg 1894;20:497-555.

4. Veronesi U, Cascinelli N, Nariani L, et al. Twentyyear follow-up of a randomized study comparing breastconserving surgery with radical mastectomy for early breast cancer. N Engl J Med 2002;347:1227-32.

5. Fisher B, Anderson S, Bryant J, et al. Twenty-year follow-up of a randomised trial comparing mastectomy, lumpectomy, and lumpectomy plus irradiation for the treatment of invasive breast cancer. N Engl J Med 2002;347:1233-41.

6. Rosen PP, Fracchia AA, Urban JA, et al. "Residual" mammary carcinoma following simulated partial mastectomy. Cancer 1975;35:739-47.

7. Holland R, Veling SHJ, Mravunac M, et al. Multifocality of Tis, T1-2 breast carcinomas: Implications for clinical trials of Breast-Conserving Surgery. Cancer 1985;56:979-90.

8. Hwang ES, Lichtensztajn DY, Gomez SL, et al. Survival after lumpectomy and mastectomy for early stage invasive breast cancer: the effect of age and hormone receptor status. Cancer 2013;119:1402-11

9. Agarwal S, Pappas L, Neumayer L, et al. Effect of Breast Conservation Therapy vs Mastectomy on Disease-Specific Survival for Early-Stage Breast Cancer. JAMA Surg 2014;149:267-74.

10. Hofvind S, Holen A, Aas T, et al. Women treated with breast conserving surgery do better than those with mastectomy independent of detection mode, prognostic and predictive tumour characteristics. Eur J Surg Oncol 2015;41:1417-22.
11. Hartmann-Johnsen OJ, Karesen R, Schlichting E, et al. Survival is better after breast conserving therapy than mastectomy for early stage breast cancer: A registry-based follow-up study of Norwegian women primary operated between 1998-2008. Ann Surg Oncol 2015;22:3836-45.

12. van Maaren MC, de Munck L, de Bock GH et al. 10 year survival after breast-conserving surgery plus radiotherapy compared with mastectomy in early breast cancer in the Netherlands: a population-based study. Lancet Oncol 2016;17:1158-70.

13. van der Heiden-van der Loo M, Siesling S, Wouters MWJM, et al. The value of ipsilateral breast tumour recurrence as a quality indicator: hospital variation in the Netherlands. Ann Surg Oncol 2015;22 Suppl 3:S522-8.

14. van Hezewijk M, Bastiaannet E, Putter $\mathrm{H}$ et al. Effect of local therapy on locoregional recurrence in postmenopausal women with breast cancer in the Tamoxifen Exemestane Adjuvant Multinational (TEAM) trial. Radiother Oncol 2013;108:190-6.

15. Abdulkarim BS, Cuartero J, Hanson J, et al. Increased risk of locoregional recurrence for women with T12N0 triple-negative breast cancer treated with modified radical mastectomy without adjuvant radiation therapy compared with breast -conserving therapy. J Clin Oncol 2011;29:2852-8.

16. Gusterson BA, Stein T. Human breast development. Semin Cell Dev Biol 2012;23:567-73.

17. Lemaine V, Simmons PS. The adolescent female: breast and reproductive embryology and anatomy. Clin Anat 2013:26:22-8

18. Richert MM, Schwertfeger KL, Ryder JW, et al. An atlas of mouse mammary gland development. J Mammary Gland Biol Neoplasia 2000;5:227-41.

19. Musumeci G, Castrogionanni P, Szychlinska MA, et al. Mammary gland: fromembryongenesis to adult life. Acta Histochem 2015;117:379-85.

20. Moffat DF, Going JJ. Three dimensional anatomy of complete duct systems in human breast: pathological and developmental implications. J Clin Pathol 1996;49:48-52.

21. Tot T. DCIS, cytokeratins, and the theory of the sick lobe. Virchows Arch 2005;447:1-8.

22. Agelopoulos K, Buerger H, Brandt B. Allelic imbalances of the egfr gene as key events in breast cancer progression - the concept of committed progenitor cells. Curr Cancer Drug Targets 2008;8:431-45.

23. Tot T, Gere M. Radiologically unifocal invasive breast carcinomas: large-section histopathology correlate and impact on surgical management. J Cancer Sci Ther 
2016;8:50-4.

24. Going JJ, Mohun TJ. Human breast duct anatomy, the 'sick lobe' hypothesis and intraductal approaches to breast cancer. Breast Cancer Res Treat 2006;97:285-91.

25. Tot $\mathrm{T}$. The role of large-format histopathology in assessing subgross morphological prognostic parameters: a single institution report of 1000 consecutive breast cancer cases. Int J Breast Cancer 2012;2012:395415.

26. Tot T. Early and more advanced unifocal and multifocal breast carcinomas and their molecular phenotypes. Clin Breast Cancer 2011;11:258-63.

27. Lopez-Garcia MA, Geyer FC, Lacroix-Triki M, et al. Breast cancer precursors revisited: molecular features and progression pathways. Histopathology 2010;57:171-92.

28. Desmedt C, Fumagalli D, Pietri E, et al. Uncovering the genomic heterogeneity of multifocal breast cancer. J Pathol 2015;236:457-66.

29. Lebya K, Garcia-Smith R, Swaminathan R, et al. Towards a personalised surgical margin for breast conserving surgery - implications of field cancerisation in local recurrence. J Surg Oncol 2017;115:109-15.

30. Edgerton ME, Chuang YL, Yang WT, et al. A novel, patient-specific mathematical pathology approach for assessment of surgical volume: application to ductal carcinoma in situ of the breast. Anal Cell Pathol (Amst) 2011;34:247-63.

31. Amy D, Durante E, Tot T. The lobar approach to breast ultrasound imaging and surgery. J Med Ultrason (2001) 2015;42:331-9.

32. Mitchell SD. A step-by-step oncoplastic breast conservation surgical atlas of reproducible dissection techniques and anatomically ideal incision placement. Breast Cancer Res Treat 2017;165:505-16.

33. Tan M, Ung O. Alternative approaches for oncoplastic breast surgery. Ann Surg Oncol 2011;18:297-9.

34. Mattingly AE, Ma Z, Smith PD, et al. Early postoperative complications after oncoplastic reduction. South Med J 2017;110:660-6.

35. Weber WP, Soysal SD, Zeindler J, et al. Current standards in oncoplastic breast conserving surgery. Breast 2017;34 Suppl 1:S78-81.

Cite this article as: Tan MP, Tot T. The sick lobe hypothesis, field cancerisation and the new era of precision breast surgery. Gland Surg 2018;7(6):611-618. doi: 10.21037/gs.2018.09.08
36. Scott JG, Berglund A, Schell MJ, et al. A genomebased model for adjusting radiotherapy dose (GARD): a retrospective, cohort-based study. Lancet Oncol 2017;18:202-11.

37. Tan MP. A novel segment classification for multifocal and multicentric breast cancer to facilitate breast-conservation treatment. Breast J 2015;21:410-7.

38. Sun Y, Liao M, He L, et al. Comparison of breastconserving surgery with mastectomy in locally advanced breast cancer advanced breast cancer after good response to neoadjuvant chemotherapy. Medicine (Baltimore) 2017;96:e8367.

39. Lang Z, Wu Y, Li C, et al. Multifocal and multicentric breast carcinoma: a significantly more aggressive tumour than unifocal breast cancer. Anticancer Res 2017;37:4593-8.

40. Tot T, Gere M, Pekar G, et al. Breast cancer multifocality, disease extent and survival. Hum Pathol 2011;42:1761-9.

41. Pekar G, Gere M, Tarjan M, et al. Molecular phenotype of the foci in multifocal invasive breast carcinomas. Cancer 2014;120:26-34.

42. Blighe K, Kenny L, Patel N, et al. Whole genome sequence analysis suggests intratumoral heterogeneity in dissemination of breast cancer to lymph nodes. PLoS One 2014;9:e115346.

43. Gentilini OD, Cardoso MJ, Poortmans P. Less is more: breast conservation might be even better than mastectomy in early breast cancer patients. Breast 2017;35:32-3.

44. MacNeill F, Karakatsanis A. Over surgery in breast cancer. Breast 2017;31:284-9.

45. Tan MP, Ong EM, Amy D, et al. Integrating Anatomy, Radiology, Pathology, and Surgery: An Alternative Approach in Resecting Multifocal and Multicentric Breast Carcinoma. Breast J 2017;23:663-9.

46. Dushyanthen S, Beavis PA, Savas P, et al. Relevance of tumour-infiltrating lymphocytes in breast cancer. BMC Medicine 2015;13:202.

47. Lumachi F, Chiara GB, Foltran L, et al. Proteomics as a guide for personalised adjuvant chemotherapy in patients with early breast cancer. Cancer Genomics Proteomics 2015;12:385-90. 